\title{
Accounting for variable operation conditions when optimizing cogeneration GTU and CCGT
}

\author{
Aleksandr M. Kler ${ }^{1}$, Pavel V. Zharkov ${ }^{1}$, Elena L. Stepanova ${ }^{1}$ and Yulia M. Potanina ${ }^{1, *}$ \\ ${ }^{1}$ Melentiev Energy Systems Institute of Siberian Branch of the Russian Academy of Sciences, 130 Lermontov str., Irkutsk, Russia
}

\begin{abstract}
The paper deals with a new approach to mathematical modeling and optimization of cogeneration GTU and CCGT, taking into account the variable conditions of their work, developed at the ISEM SB RAS. An analysis was carried out of the features of using this approach in relation to the thermal power plants. According to this approach at mathematical modelling of the plant there are structural optimized parameters (affecting the design of the plant), mode optimized parameters (affecting the operation of the plant in the mode with partial thermal loads) and balancing parameters affecting solution of the system of equations in the nominal mode and in modes at partial loads The connection between the design calculation (at rated loads) and verification calculations (at partial thermal loads) is carried out through the structural characteristics of the plant elements, determined during the design calculation. Taking into account these features, the problem of optimization of continuously changing parameters of cogeneration TPP was formulated, focused on the use of the developed optimization method. New mathematical models of the elements of the thermal power plants have been created. Optimization technical and economic studies of the considered plants were carried out in relation to various climatic conditions.
\end{abstract}

\section{Introduction}

A significant part of the world's electrical energy is produced by thermal power plants (TPPs) that burn fossil fuels. A significant number of such plants operate with variable external conditions. This concerns changes in the heat loads of heating units that carry out combined heat and power generation, due to a change in the heating load proportional to the outside temperature. In their mathematical modeling, it is necessary to take into account several characteristic operating modes. In this case, in one typical mode (most often in a mode with rated loads), the so-called design calculation of the plant is carried out. With the given parameters of the thermodynamic cycle and some design parameters of the plant elements, the calculation of all missing design and other parameters is carried out, which makes it possible to determine the investment to the plant, the energy consumption for auxiliary needs, etc. This mode can be named as a design mode, because it defines the design characteristics. In other typical modes, with fully known design parameters of all elements, the so-called verification calculation is carried out, which allows, for given design characteristics of the TPP elements and external conditions, to determine the flow rates of working fluids and coolants and thermodynamic parameters at all points of the TPP technological scheme. It should be noted that we are talking about the calculations of the operating modes established in time. Both of these types of calculations are reduced to solving

*orresponding author: julia@,isem.irk.ru nonlinear systems of algebraic and transcendental equations.

Optimization of TPP taking into account variable operating modes was considered in several works of ISEM SB RAS [1-4]. Optimization problems were formulated, and optimization calculations were carried out for cogeneration steam turbine, gas turbine and combined cycle plants using gradient methods that allow optimizing a large number of parameters. However, the cumbersomeness of the coordinated calculation of several modes greatly increased the optimization time and significantly worsened the convergence of the optimization process. Significant problems arose when using gradient optimization methods, since the determination of gradients due to the complexity of mathematical models of thermoelectric power plants is possible only by the finite-difference method. When considering several modes of operation, the errors of the verification calculations were superimposed on the errors of the design calculation, which significantly reduced the accuracy of determining the gradients of the objective function and restrictions. This significantly reduced the accuracy of finding the optimal solution and the stability of the optimization process.

The indicated optimization problems, taking into account several characteristic modes of operation of the TPP, are inherent in the traditional approach to mathematical modeling and optimization of the TPP parameters. According to this approach, mathematical models of elements (heat exchangers, turbomachines, combustion chambers, etc.) are developed. With each 
reference to such a model, a nonlinear system of equations is solved that describes the corresponding element. As a rule, the calculation is performed by the iterative method. The calculation of the plant as a whole is reduced to an iterative process (using the same methods), at each iteration of which the models of the plant elements are sequentially addressed and the systems of equations for these elements are solved.

Since all the indicated systems of equations are nonlinear, their exact solution is impossible, which leads to the previously noted errors. At the same time, an increase in the accuracy of solving systems leads to a significant increase in the time of optimization calculations.

In [5], a new effective approach to mathematical modeling and optimization of TPP was proposed. In accordance with this approach, the process of solving nonlinear systems of equations of elements and the plant as a whole is transferred from the level of mathematical modeling to the level of optimization. In this case, all iterative processes are removed from the models of the plant elements and only the calculations of the residuals of the corresponding equations are left. This gives a dramatic improvement in the speed, convergence and stability of the optimization process, as well as an increase in the accuracy of finding the optimal solution.

In this paper, the features of this approach are considered when taking into account variable operating conditions.

The problem of optimizing the TPP parameters according to the criterion of economic efficiency (electricity price, heat price and internal rate of return on investment), taking into account several operating modes, according to a new approach to mathematical modeling of TPP, can be formulated as follows (problem I).

Find

$$
\begin{gathered}
\min C^{e l}\left(I R R, E_{y r}, Q_{y r}, B_{y r}, K, d^{e c}\right)(1) \\
x^{n m}, y^{p t}, x_{1}^{p t}, y_{1}^{p t}, \ldots, x_{n^{x}}^{p t}, y_{n^{x}}^{p t}
\end{gathered}
$$

subject to

$$
\begin{aligned}
& \theta^{n m}=H^{n m}\left(x^{n m}, y^{n m}, d^{n m}\right), \\
& -z_{T P} \leq \frac{\theta_{j}^{n m}}{\varepsilon_{j}^{n m}} \leq z_{T P}, j=1, \ldots, L^{n m} \\
& G^{n m}\left(x^{n m}, y^{n m}, d^{c}\right) \geq 0, \\
& \underline{x}^{n m} \leq x^{n m} \leq \bar{x}^{n m}, \\
& S^{K}=\varphi^{n m}\left(x^{n m}, y^{n m}, d^{n m}\right), \\
& K=\psi\left(S^{K}, d^{n m}\right), \\
& B^{n m}=B^{n m}\left(x^{n m}, y^{n m}, d^{n m}\right), \\
& Q^{n m}=Q^{n m}\left(x^{n m}, y^{n m}, d^{n m}\right), \\
& N^{n m}=N^{n m}\left(x^{n m}, y^{n m}, d^{n m}\right), \\
& \theta_{i}^{p t}=H^{p t}\left(x_{i}^{p t}, y_{i}^{p t}, S^{K}, d_{i}^{p t}\right),
\end{aligned}
$$

$$
\begin{aligned}
& -z_{T P} \leq \frac{\theta_{j}^{p t}}{\varepsilon_{j}^{p t}} \leq z_{T P}, j=1, \ldots, L^{p t} \\
& G_{i}^{p t}\left(x_{i}^{p t}, y_{i}^{p t}, S^{K}, d_{i}^{p t}\right) \geq 0, \\
& \underline{x}^{p t} \leq x_{i}^{p t} \leq \bar{x} p \\
& B_{i}^{p t}=B^{p t}\left(x_{i}^{p t}, y_{i}^{p t}, S^{K}, d_{i}^{p t}\right), \\
& N_{i}^{p t}=N^{p t}\left(x_{i}^{p t}, y_{i}^{p t}, S^{K}, d_{i}^{p t}\right), \\
& Q_{i}^{p t}=Q^{p t}\left(x_{i}^{p t}, y_{i}^{p t}, S^{K}, d_{i}^{p t}\right), \\
& i=1, \ldots, n^{X}, \\
& E_{y r}=N^{n m} \tau^{n m}+\sum_{i=1}^{n^{X}} N_{i}^{p t} \tau_{i}^{p t}, \\
& Q_{y r}=Q^{n m} \tau^{n m}+\sum_{i=1}^{n^{X}} Q_{i}^{p t} \tau_{i}^{p t}, \\
& B_{y r}=B^{n m} \tau^{n m}+\sum_{i=1}^{n^{X}} B_{i}^{p t} \tau_{i}^{p t},
\end{aligned}
$$

where $C^{e l}$ is the the price of electricity determined at a given internal rate of return on investment and the price of heat supplied from the TPP, $E_{y r}$ is the annual electric power supply, $Q_{y r}$ is the annual heat energy supply, $B_{y r}$ is the annual fuel consumption, $K$ - capital investments into the plant, $d^{e c}$ - initial economic data required to determine $C^{e l}$ (heat price, fuel price, share of conditionally fixed costs from capital investments, etc.), $H^{n m}-L^{n m}$-th dimensional system of nonlinear algebraic and transcendental equations describing design calculations in nominal (design) mode, $\theta^{n m}-L^{n m}$-th dimensional vector of residuals of the system of equations $H^{n m}, x^{n m}-n^{n m}$-th dimensional vector of optimized parameters in the design mode (flow rate of working fluides, parameters of the thermodynamic cycle, structural parameters of elements), $y^{n m}-L^{n m}$ dimensional vector of calculated balanced parameters responsible for the solution of the system $H^{n m}, d^{n m}$ vector of constant parameters used in calculating the design mode, $\underline{x}^{n m}, \bar{x}^{n m}$ - vectors whose components define the minimum and maximum bounds for the corresponding component of the vector $x^{n m}, G^{n m}$ $m^{n m}$-th dimensional system of inequality constraints, which determines the range of admissible values of parameters in the design mode, $S^{K}$ is the vector of design parameters of the plant determined at design mode, $d^{c}$ is the vector of costs of the equipment components, $B^{n m}, Q^{n m}, N^{n m}$ - hourly consumption of equivalent fuel, hourly heat supply to consumers and useful electric power in design mode, respectively, $H^{p t}$ 
- $L^{p t}$-th dimensional system of nonlinear algebraic and transcendental equations describing modes with offdesign operating conditions, $\theta_{i}^{p t}-L^{p t}$-th dimensional vector of $H^{p t}$-th system residuals in the $i$-th off-design mode at partial loads, $x_{i}^{p t}-n^{p t}$ - dimensional vector of the optimized parameters in the $i$-th off-design mode at partial loads (fuel consumption, pressure in the regulated steam extraction of a cogeneration steam turbine, etc.), $y_{i}^{p t}-L^{p t}$ - dimensional vector of calculated balanced parameters responsible for the solution of the system $H^{p t}$ at the $i$-th mode at partial loads, $d_{i}^{p t}$ - vector of constant parameters used in the $i$-th off-design mode at partial loads, $G_{i}^{p t}-m^{p t}$-th dimensional system of constraints - inequalities, which determines the range of admissible values of parameters in the $i$-th off-design mode at partial loads, $\underline{x}^{p t}, \bar{x}^{p t}$ - vectors whose components define the minimum and maximum bounds for the corresponding components of the vectors $x_{i}^{p t}$, $B_{i}^{p t}, N_{i}^{p t}, Q_{i}^{p t}$ - hourly consumption of equivalent fuel, useful electric power and hourly heat supply in the $i$-th off-design mode at partial loads, $n^{X}$ - the number of modes at partial loads with carrying out verification calculations, $\tau^{n m}$ is the annual duration of the design mode, $\tau_{i}^{n m}$ is the annual duration of the $i$-th off-design mode at partial loads, $\varepsilon_{j}^{n m}$ is the required accuracy of the solution at the $j$-th level of the system $H^{n m}, \varepsilon_{j}^{p t}$ is the required accuracy of solving the $j$-th equation of the system $H^{n m}, z_{T P}$ - constraints on relative residuals modules $\frac{\theta_{j}^{n m}}{\varepsilon_{j}^{n m}}$ and $\frac{\theta_{j}^{p t}}{\varepsilon_{j}^{p t}}$.

The approach to optimization of TPP parameters considered in [5] in relation to problem (I) has the following form. In expressions (3) and (12) $z_{T P}$ is replaced by the parameter $z$. Each iteration takes two steps. At the first step, problem (I) is solved (taking into account the indicated changes). Moreover, at the first iteration, $z$ takes on a large value $z^{0}>>1$. It should be noted that $z$ is fixed at the first step.

At the second step, $\mathrm{z}$ is added to the optimized parameters and the problem of minimizing $z$ is solved under all conditions (8)-(21) and with an additional condition $z \geq \frac{F(x, y)-F^{t}}{\sigma}$, where $\sigma$ is the required accuracy of determining the optimal solution, $F^{t}$ is the value of the objective function at the point of solving problem (I) at the first step. When solving the problem of the first step at the current iteration, the optimal value $z^{t}$ obtained at the second step of the previous iteration is taken as a fixed value $z$. This process continues until the condition $z^{t} \leq z^{r}$ is met, where $z^{r}$ is the required value of $z$.

Analysis of the structure of relations between the parameters of design and verification calculations shows that a change in any component of vectors $x^{n m}$ and $y^{n m}$, with constant values of all components of vectors $y_{i}^{n m}$ and $y^{n m}$, will affect the result of all verification calculations. This is due to the connection on the vector $S^{K}$. Therefore, among partial derivatives of the form $\frac{\partial g_{i a}^{p t}}{\partial x_{j}^{n m}}, \frac{\partial \theta_{i b}^{p t}}{\partial x_{j}^{n m}}, \frac{\partial Q_{i}^{p t}}{\partial x_{j}^{n m}}, \frac{\partial N_{i}^{p t}}{\partial x_{j}^{n m}}, \frac{\partial B_{i}^{p t}}{\partial x_{j}^{n m}}$ and $\frac{\partial g_{i a}^{p t}}{\partial y_{l}^{n m}}$, $\frac{\partial \theta_{i b}^{p t}}{\partial y_{l}^{n m}}, \frac{\partial Q_{i}^{p t}}{\partial y_{l}^{n m}}, \frac{\partial N_{i}^{p t}}{\partial y_{l}^{n m}}, \frac{\partial B_{i}^{p t}}{\partial y_{l}^{n m}}$, there will be non-zero elements. This, in turn, requires determining partial derivatives of software and performing both design and all verification calculations. This, in turn, requires the performance of both design and all verification calculations when determining the partial derivatives with respect to $x_{j}^{n m}$ and $y_{l}^{n m}$. On the contrary, changing the components of the vectors $x_{i}^{p t}$ and $y_{i}^{p t}$ with the values of $x^{n m}$ and $y^{n m}$ unchanged and the parameters of $x_{j}^{p t}$ will only change the results of the i-th verification calculation. Therefore, the partial derivatives of all functions, except for the derivatives of vector functions $\theta_{i}^{p t}, g_{i}^{p t}$, and scalar functions $B_{i}^{p t}, N_{i}^{p t}$, $Q_{i}^{p t}$ will be equal to zero. Thus, when determining the partial derivatives with respect to the components of the vectors $x_{i}^{p t}$ and $y_{i}^{p t}$, it is sufficient to carry out a verification calculation of this mode only. This makes it possible to reduce significantly the amount of computations when determining derivatives using the finite-difference method.

An important means of speeding up optimization calculations is the use of parallel calculations. In the optimization algorithm used in this work, there are two stages where such an application can have a significant effect. This primarily refers to the calculation of the objective function gradients and constraint gradients by the finite-difference method. The calculation of the partial derivative of some function $f$ with respect to the $i$ th component of some vector $x_{i}$ is carried out by the expression for the central difference of the form $\frac{\partial f}{\partial x_{i}} \approx \frac{f\left(x_{1}^{0}, \ldots x_{i-1}^{0}, x_{i}^{0}+\Delta x_{i}, x_{i+1}^{0}, \ldots x_{b}^{0}\right)-f\left(x_{1}^{0}, \ldots, x_{i-1}^{0}, x_{i}^{0}-\Delta x_{i}, x_{i+1}^{0}, \ldots x_{n}^{0}\right)}{2 \cdot \Delta x_{i}}$ where $x_{j}^{0}, \ldots j=1, \ldots, n$ are the values of the $j$-th component of the vector $x$ at the base point, $\Delta x_{i}$ is a trial step along the $i$-th component of the vector $x$. After the base point $x^{0}$ is known, the determination of partial 
derivatives of an arbitrary function $f$ over all components of the vector $x$ can occur independently, and two calculations of the function $f$ must be performed to determine one partial derivative. In one calculation, the $i$-th parameter of the vector $x$ takes the value $x_{i}^{0}+\Delta x_{i}$, and in the other calculation $-x_{i}^{0}-\Delta x_{i}$. In relation to the considered TPPs optimization, the derivatives are found over the components of independent vectors $x^{n m}, y^{n m}, x_{i}^{n m}, y_{i}^{n m}, i=1, \ldots n_{r e}$. Given the dimension of these vectors, the calculation of partial derivatives can be divided into $n^{n m}+L^{n m}+n^{X}\left(n^{n m}+L^{n m}\right)$ parallel branches. . At the same time, two calculations must be performed on each branch, including the determination of all residuals and inequality constraints, as well as the objective function. Taking into account the above-mentioned feature of the relationship between the design and verification calculations, both design and verification calculations are performed when determining derivatives from the components of vectors $x^{n m}$ and $y^{n m}$. When determining derivatives from the components of vectors $x_{i}^{p t}$ and $y_{i}^{p t}$, only the $i$-th verification calculation is performed.

Another stage of the optimization algorithm, which allows organizing parallel computations, is onedimensional minimization along the chosen direction. It should be noted that the methods of half division, tangents and their combinations often used at this stage do not allow parallel calculations, since the choice of the current point along the direction of descent depends on the choice of the point in the previous step.

In this paper, we use a one-dimensional minimization method that does not have this drawback. After the descent direction $S$ is selected from the point $x^{0}$, any point $x$ along this direction is determined from the expression $x=x^{0}+\lambda \cdot S$, where $\lambda$ is a positive scalar value (step along the direction $S$ ). Since the interior point method is used for optimization, the condition $\psi\left(x^{0}\right)>0$ is always satisfied, where $\psi$ is a vector function of all constraints - inequalities of the problem. By linearizing the vector function $\psi$ at the point $x^{0}$, it is easy to determine the maximum step $\lambda^{\max }$ for meeting the condition $\psi\left(x^{0}\right) \geq 0$. The value $\Delta \lambda=\frac{\lambda_{\max }}{n+1}$ is determined and $n$-steps $\lambda_{i}=\Delta \lambda \cdot i$, $i=1, \ldots, n$ are found. The coordinates of the points $x=x^{0}+\lambda \cdot S, \quad i=1, \ldots, n$ are determined. At all points $x_{i}$, design and verification calculations of the plant are carried out. The search is performed for a valid point where all constraints are met as strict inequalities, and the objective function reaches the lowest value among $n$ points. Calculations of all $n$ points are independent and can be performed on parallel branches.
It should be noted that in order to transfer the solution of systems of equations from the level of mathematical models, it is necessary to remove from them all computational cycles used to organize iterative processes. In the element model, only the residuals of its system of equations are calculated, which are the information-output parameters of the model. The variables that, in the traditional construction of the model, were determined as a result of solving the system of equations (balancing variables) are the informationinput parameters of the model. Changing these variables allows you to reduce the values of the residuals modules to the required level. As already noted, according to the developed optimization method, the balancing variables act as additional optimized parameters. On the basis of these principles, mathematical models of the elements of technological schemes presented below were developed.

\section{Optimization of operating modes of cogeneration gas turbine and combined cycle power plants}

\subsection{Optimization studies of the cogeneration gas turbine unit}

Optimization studies of a cogeneration gas turbine unit (GTU) (Fig. 1) were carried out for two variants of climatic conditions. One nominal mode (design calculation), three modes at partial heat loads and one mode at a load on hot water supply only (non-heating period) were considered, in which verification calculations were carried out.

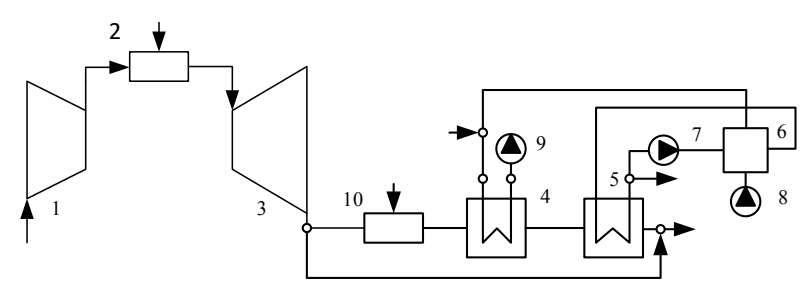

Fig: 1. Design flow diagram of the cogeneration gas turbine unit.

1 - air compressor, 2 - combustion chamber, 3 - gas turbine, 4 - waste heat boiler, 5 - contact heat exchanger, 6 - water heater, 7-9 - pumps, 10 - additional combustion chamber.

As an optimization criterion when conducting optimization studies, the price of electricity was taken at a given price of supplied heat and an internal rate of return on investment, which determines the level of economic efficiency of the investment project, and also the optimized parameters of the cycle in the nominal mode, design and mode optimized parameters, the number of which for each gas turbine had 11, 12 and 25 parameters, respectively. In the design mode, the list of parameters to be optimized included: the calculated heat load of the consumer; fuel consumption; internal, external diameters and pipe pitches of the heating surfaces of the waste heat boiler and heat exchangers; mass water velocities; gas pressure at the outlet of the air compressor; gas temperature at the gas turbine inlet; the 
share of the flow rate of exhaust gases from their total flow after the gas turbine going to the waste heat boiler. As regime optimized parameters in characteristic modes (verification calculations) were taken: fuel consumption; heating water consumption of the heating water make-up heater; gas temperature at the gas turbine inlet; the share of the flow rate of exhaust gases from their total flow after the gas turbine going to the waste heat boiler. Restrictions were imposed on the maximum permissible gas temperature at the gas turbine inlet, the nonnegativity of the temperature head in the waste heat boiler, and on the mechanical stresses of the metal of the heat exchanger pipes. The number of imposed restrictions for power plants in each of the regions under consideration is 268 parameters.

Optimization calculations were carried out under the following conditions. The range of variation of the electrical power of the gas turbine was taken equal to 50$60 \mathrm{MW}$. The design outdoor temperature for the heating system for option 1 was taken as $-25^{\circ} \mathrm{C}$, for option 2 it was $-55^{\circ} \mathrm{C}$, the temperature chart of the heating network for option 1 was taken as $120 / 70{ }^{\circ} \mathrm{C}$, for option 2 it was $150 / 70{ }^{\circ} \mathrm{C}$. The heating period was divided into three intervals. For option 1, the duration of heating periods was 418 hours, 1316 hours, 3176 hours, the design outdoor temperature during these periods: $20^{\circ} \mathrm{C},-10{ }^{\circ} \mathrm{C}$, $1.5{ }^{\circ} \mathrm{C}$, respectively. For option 2 , the duration of the heating periods was 1617 hours, 2262 hours, 2217 hours, the design outdoor temperature during these periods: -40 ${ }^{\circ} \mathrm{C},-20{ }^{\circ} \mathrm{C}, 2{ }^{\circ} \mathrm{C}$, respectively. The durations of nonheating periods were taken as 3850 hours and 2664 hours. The maximum permissible value of the gas temperature in front of the gas turbine is $1450^{\circ} \mathrm{C}$. When calculating the capital investments of the GTU, the following initial information was taken. Specific costs: gas turbine -70 dollars $/ \mathrm{kW}$ (per unit of maximum full power of the gas turbine), air compressor -50 dollars $/ \mathrm{kW}$, turbine electric generator, pumps - 60 dollars $/ \mathrm{kW}$, heat exchangers made of carbon steel -7000 dollars/ton, from steel 20000 - 21000 dollars/ton, electrical equipment 192 dollars/kW, systems depending on fuel consumption 240000 dollars/(ton/h). The internal rate of return on capital investments is 0.15 , the coefficient of accounting for construction costs is 1.6; coefficient taking into account unforeseen costs -1.03 ; coefficient taking into account other costs -1.3 ; the coefficient of price adjustment for equipment -1.65 ; coefficient taking into account the cost of unaccounted equipment -1.1 . The coefficient of the increase in the cost of construction in the climatic conditions of option 2 is taken equal to 1.52 . The studies were carried out at fuel prices: for option $1-100$ dollars/tce, for option 2 175 dollars/tce.

The main annual indicators for calculating the modes of the cogeneration gas turbine plant are given in table 1 .

Table 1. The main annual indicators of the GTU

\begin{tabular}{|l|c|c|}
\hline \multirow{2}{*}{ Main indicators } & \multicolumn{2}{c|}{ Options } \\
\cline { 2 - 3 } & 1 & 2 \\
\hline Price for heat energy, dollars/Gcal & 20,0 & 30,0 \\
\hline Price for electricity, cents/kW & 3,05 & 5,44 \\
\hline
\end{tabular}

\begin{tabular}{|l|c|c|}
\hline Specific investments, dollars/kW & 537,8 & 774,2 \\
\hline $\begin{array}{l}\text { Annual fuel consumption, thousands } \\
\text { ton ce }\end{array}$ & 187,7 & 183,5 \\
\hline $\begin{array}{l}\text { Annual power consumption, } \\
\text { thousands MWh }\end{array}$ & 468,18 & 480,2 \\
\hline $\begin{array}{l}\text { Annual heat consumption, thousands } \\
\text { Gcal }\end{array}$ & 608,1 & 590,0 \\
\hline Utilization ratio of heat fuel & 0,77 & 0,78 \\
\hline $\begin{array}{l}\text { Specific consumption of conventional } \\
\text { fuel for heat release, kg ce/ Gcal }\end{array}$ & 153,6 & 153,6 \\
\hline $\begin{array}{l}\text { Specific consumption of conventional } \\
\text { fuel for electricity supply, g ce/kWh }\end{array}$ & 201,4 & 193,6 \\
\hline $\begin{array}{l}\text { Annual fuel consumption of the } 1 \mathrm{st} \\
\text { combustion chamber of the gas } \\
\text { turbine, thousands ton ce }\end{array}$ & 166,3 & 167,4 \\
\hline $\begin{array}{l}\text { Annual fuel consumption of the } 2 \mathrm{nd} \\
\text { combustion chamber of the gas } \\
\text { turbine, thousands ton ce }\end{array}$ & 21,4 & 16,1 \\
\hline
\end{tabular}

Analysis of the results of optimization studies of GTU operating modes in both regions showed the following. The heat load of power plants is covered by heating the network water in the heat recovery boiler without attracting a peak source. With the fuel and heat prices taken into account, the electricity price was: for option $1-3.05$ cents/kWh, for option $2-5.44$ cents/ $\mathrm{kWh}$, respectively. The coefficient of fuel heat utilization is equal to: for option $1-0.77$, for option $2-$ 0.78 ; the specific investment for option $1-537.8$ dollars/kW, option $2-774.2$ dollars/kW. The cost of conventional fuel for electricity supply: for option 1 $201.4 \mathrm{~g}$ ce $/ \mathrm{kWh}$, for option $2-193.6 \mathrm{~g}$ ce $/ \mathrm{kWh}$. The maximum useful electrical power from both the gas turbine in design operating mode reaches $60 \mathrm{MW}$.

\subsection{Optimization studies of cogeneration combined cycle power plant}

The application of the new approach to the modeling of power plants is presented by the example of optimization of the parameters of the cogeneration combined cycle power plant, the diagram of which is shown in Fig. 2.

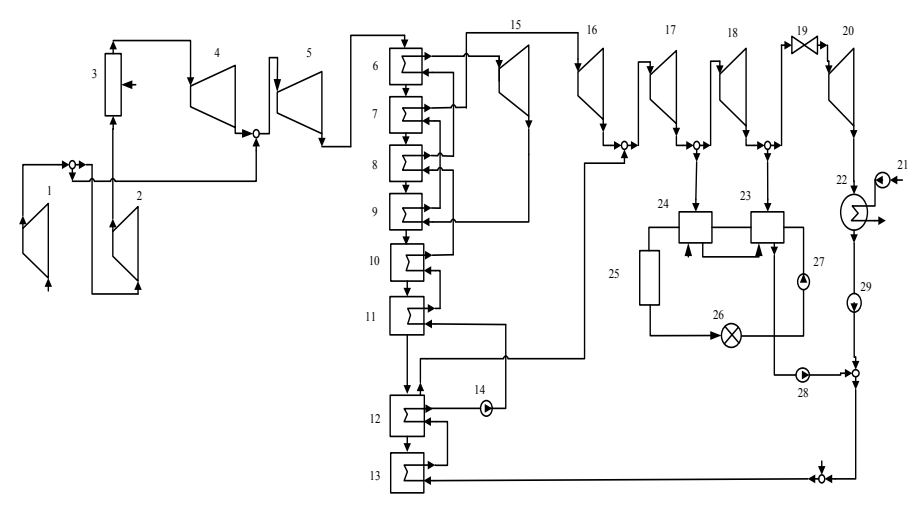

Fig: 2. Design flow diagram of the cogeneration CCGT. 1,2 - air compressors, 3 - combustion chamber of a gas turbine, 4, 5 - sections of a gas turbine, 6,8 - stages of a convective steam reheater, 7,9 - stages of an intermediate 
steam reheater, 10, 12 - high and low pressure evaporators, 11,13 - water economizer stages, 14 - feed water pump, 1518,20 - steam turbine compartments, 19 - regulating diaphragm, 21 - circulation pump, 22 - condenser, $23.24-$ network heaters, 25 - peaking water boilers, 26 - heat consumer, 27,28,29- pumps.

The plant includes a gas turbine unit with air extraction from the compressor for cooling the nozzle and rotor blades of the GT, a waste-heat boiler using combustion products, a cogeneration steam turbine, and a peak hot water boiler.

The mathematical model of the plant as a whole includes new mathematical models (without iterative cycles) of its elements - gas turbines, air compressors, convective gas-water heat exchangers on combustion products, sections of a steam turbine, and a condenser. The following parameters were optimized: heat load of the consumer in the design mode, fuel consumption into the combustion chamber, diameters, pipe pitches of heat exchangers of the waste heat boiler, mass velocities of water (steam) in them, enthalpy of water (steam) at the outlet, width and depth of the flue, pressure and enthalpy of live steam, circulation ratio in circuits with drums separators, etc. The limitations that determine the area of physically and technically permissible operation of the plant: on mechanical stresses and the limiting temperature of the metal of heat exchanger pipes, nonnegativity of temperature head at the gas inlet and outlet, flow rates of working fluids etc. were taken into account. The general calculation model contains 487 optimized and balancing parameters (131 in the design calculation and 89 in each verification calculation) and 589 inequality constraints (112 in the design calculation and 118 in each verification calculation).

As an efficiency criterion for optimization, the price of electricity was used at a given internal rate of return on investment. The design outdoor temperatures and the duration of the heating periods are taken the same as in the optimization calculations of the GTU. The results of optimization calculations are presented in Table 2 (flow rates, pressures and temperatures at various points of the flowchart are given for the mode with the rated load).

Table 2. Technical and economic indicators of TPP

\begin{tabular}{|l|c|c|}
\hline \multicolumn{1}{|c|}{ Main indicators } & \multicolumn{2}{|c|}{ Value } \\
\hline $\begin{array}{l}\text { Gas temperature at the exit of the } \\
\text { combustion chamber, }{ }^{\circ} \mathrm{C}\end{array}$ & 1300 & 1300 \\
\hline $\begin{array}{l}\text { Gas temperature at the inlet of the } \\
\text { heat recovery boiler, }{ }^{\circ} \mathrm{C}\end{array}$ & 693 & 689 \\
\hline Temperature of live steam, ${ }^{\circ} \mathrm{C}$ & 488 & 504 \\
\hline Pressure of live steam, $\mathrm{MPa}$ & 13,0 & 13,8 \\
\hline Consumption of live steam, $\mathrm{kg} / \mathrm{s}$ & 80,4 & 90,5 \\
\hline Temperature of reheat steam, ${ }^{\circ} \mathrm{C}$ & 472 & 490 \\
\hline Pressure of reheat steam, $\mathrm{MPa}$ & 3,5 & 3,6 \\
\hline Consumption of reheat steam, $\mathrm{kg} / \mathrm{s}$ & 80,4 & 90,5 \\
\hline $\begin{array}{l}\text { Annual fuel } \\
\text { thousands ton ce }\end{array}$ & 450,6 & 557,6 \\
\hline Steam pressure in the $2 \mathrm{nd}$ heater, & 0,09 & 0,11 \\
\hline
\end{tabular}

\begin{tabular}{|l|c|c|}
\hline $\mathrm{MPa}$ & & \\
\hline $\begin{array}{l}\text { Steam pressure in the 1st heater, } \\
\mathrm{MPa}\end{array}$ & 0,06 & 0,078 \\
\hline $\begin{array}{l}\text { Steam pressure in the condenser, } \\
\mathrm{MPa}\end{array}$ & 0,0038 & 0,0037 \\
\hline Calculated heat load, Gcal/h & 376,2 & 351,7 \\
\hline Capacity, MW & 353 & 364,4 \\
\hline Price for electricity, cents/kWh & 8,09 & 8,64 \\
\hline Specific investments, dollars/kW & 1596 & 1603 \\
\hline Extraction factor & 0,62 & 0,73 \\
\hline
\end{tabular}

\section{Conclusions}

Application of a new effective approach to optimization of parameters of thermal power plants operating with variable external conditions is presented. In accordance with this approach, the process of solving nonlinear systems of equations of elements and the plant as a whole is transferred from the level of mathematical modeling to the level of optimization. New mathematical models of the elements of the thermal power plants have been created, focused on performing design and verification calculations. Optimization technical and economic studies of the considered thermal power plants in various climatic conditions were carried out.

This study was carried out at Melentiev Energy Systems Institute of Siberian Branch of the Russian Academy of Science (ESI SB RAS) (Irkutsk, Russia) and supported by the grant of the Russian Science Foundation (project No. 16-1910174).

\section{References}

1. A. M. Kler, A. Yu. Marinchenko, S. N. Sushko, Thermophys. Aeromech. 13, 283-293 (2006).

2. A.M.Kler, N.P.Dekanova, T.P.Shchegoleva, et al., The Methods Of Optimizing Complex Power Plants (Novosibirsk, VO Nauka, 1993) [in Russian].

3. A. M. Kler, A. Yu. Marinchenko, Yu. M. Potanina, Therm. Eng, 56 (9) (2009).

4. A. M. Kler, Yu. M. Potanina, A. S. Maximov, Therm. Eng., 59 (7) (2012).

5. A. M. Kler, P. V. Zharkov, N. O. Epishkin, Energy, 189 (2019) 116230. 